\title{
A NOTE ON DERIVATIONS IN SEMIPRIME RINGS
}

\author{
JOSO VUKMAN AND IRENA KOSI-ULBL
}

Received 20 June 2005 and in revised form 2 October 2005

We prove in this note the following result. Let $n>1$ be an integer and let $R$ be an $n$ !torsion-free semiprime ring with identity element. Suppose that there exists an additive mapping $D: R \rightarrow R$ such that $D\left(x^{n}\right)=\sum_{j=1}^{n} x^{n-j} D(x) x^{j-1}$ is fulfilled for all $x \in R$. In this case, $D$ is a derivation. This research is motivated by the work of Bridges and Bergen (1984). Throughout, $R$ will represent an associative ring with center $Z(R)$. Given an integer $n>1$, a ring $R$ is said to be $n$-torsion-free if for $x \in R, n x=0$ implies that $x=0$. Recall that a ring $R$ is prime if for $a, b \in R, a R b=(0)$ implies that either $a=0$ or $b=0$, and is semiprime in case $a R a=(0)$ implies that $a=0$. An additive mapping $D: R \rightarrow R$ is called a derivation if $D(x y)=D(x) y+x D(y)$ holds for all pairs $x, y \in R$ and is called a Jordan derivation in case $D\left(x^{2}\right)=D(x) x+x D(x)$ is fulfilled for all $x \in R$. Every derivation is a Jordan derivation. The converse is in general not true. A classical result of Herstein (1957) asserts that any Jordan derivation on a prime ring with characteristic different from two is a derivation. A brief proof of Herstein's result can be found in 1988 by Brešar and Vukman. Cusack (1975) generalized Herstein's result to 2-torsion-free semiprime rings (see also Brešar (1988) for an alternative proof). For some other results concerning derivations on prime and semiprime rings, we refer to Brešar (1989), Vukman (2005), Vukman and Kosi-Ulbl (2005).

Any derivation $D: R \rightarrow R$, where $R$ is an arbitrary ring, satisfies the relation

$$
D\left(x^{n}\right)=\sum_{j=1}^{n} x^{n-j} D(x) x^{j-1}, \quad x \in R .
$$

It seems natural to ask under what additional assumptions the converse is true. More precisely, under what additional assumptions an additive mapping $D$, which maps a ring $R$ into itself, satisfying the relation (1) is a derivation. Bridges and Bergen [4] answered this question in case $R$ is a prime ring with an identity element and with characteristic $m$, where $m=0$ or $m n$. It is our aim in this note to prove the following result. Our methods differ from those used by Bridges and Bergen. 
3348 A note on derivations in semiprime rings

Theorem 1. Let $n>1$ be an integer and let $R$ be a $n$ !-torsion-free semiprime ring with identity element. Suppose that there exists an additive mapping $D: R \rightarrow R$ such that

$$
D\left(x^{n}\right)=\sum_{j=1}^{n} x^{n-j} D(x) x^{j-1}
$$

is fulfilled for all $x \in R$. In this case, $D$ is a derivation.

Proof. From the relation

$$
D\left(x^{n}\right)=\sum_{j=1}^{n} x^{n-j} D(x) x^{j-1}, \quad x \in R
$$

it follows immediately that $D(e)=0$, where $e$ denotes the identity element. Putting $x+c$ for $x$ in the above relation, where $c$ is any element of the center $Z(R)$ such that $D(c)=0$, we obtain

$$
\begin{aligned}
\sum_{i=0}^{n}\left(\begin{array}{c}
n \\
i
\end{array}\right) & D\left(x^{n-i} c^{i}\right) \\
= & \left(\sum_{i=0}^{n-1}\left(\begin{array}{c}
n-1 \\
i
\end{array}\right) x^{n-1-i} c^{i}\right) D(x)+\left(\begin{array}{c}
n-2 \\
i=0
\end{array}\left(\begin{array}{c}
n-2 \\
i
\end{array}\right) x^{n-2-i} c^{i}\right) D(x)(x+c) \\
& +\left(\sum_{i=0}^{n-3}\left(\begin{array}{c}
n-3 \\
i
\end{array}\right) x^{n-3-i} c^{i}\right) D(x)(x+c)^{2}+\cdots+(x+c)^{2} D(x)\left(\sum_{i=0}^{n-3}\left(\begin{array}{c}
n-3 \\
i
\end{array}\right) x^{n-3-i} c^{i}\right) \\
& +(x+c) D(x)\left(\sum_{i=0}^{n-2}\left(\begin{array}{c}
n-2 \\
i
\end{array}\right) x^{n-2-i} c^{i}\right)+D(x)\left(\sum_{i=0}^{n-1}\left(\begin{array}{c}
n-1 \\
i
\end{array}\right) x^{n-1-i} c^{i}\right), \quad x \in R .
\end{aligned}
$$

We adopt the convention that $x^{0}=e$ for all $x \in R$. Using (1) and rearranging (4) in the sense of collecting together terms involving equal number of factors of $c$, we obtain

$$
\sum_{i=1}^{n-1} f_{i}(x, c)=0, \quad x \in R,
$$

where $f_{i}(x, c)$ stands for the expression of terms involving $i$ factors of $c$. We replace $c$ by $e$, $2 e, 3 e, \ldots,(n-1) e$ in turn in (5). Expressing the resulting system of $n-1$ homogeneous equations, we see that the coefficient matrix of the system is a van der Monde matrix

$$
\left[\begin{array}{cccc}
1 & 1 & \cdots & 1 \\
2 & 2^{2} & \cdots & 2^{n-1} \\
\vdots & \vdots & \vdots & \vdots \\
n-1 & (n-1)^{2} & \cdots & (n-1)^{n-1}
\end{array}\right]
$$


Since the determinant of the matrix is different from zero, it follows that the system has only a trivial solution. In particular,

$$
\begin{aligned}
f_{n-2}(x, e)= & \left(\begin{array}{c}
n \\
n-2
\end{array}\right) D\left(x^{2}\right)-\left(\begin{array}{l}
n-1 \\
n-2
\end{array}\right) x D(x)-\left(\begin{array}{l}
n-2 \\
n-3
\end{array}\right) x D(x)-\left(\begin{array}{l}
n-2 \\
n-2
\end{array}\right) D(x) x \\
& -\left(\begin{array}{l}
n-3 \\
n-4
\end{array}\right)\left(\begin{array}{l}
2 \\
2
\end{array}\right) x D(x)-\left(\begin{array}{l}
n-3 \\
n-3
\end{array}\right)\left(\begin{array}{l}
2 \\
1
\end{array}\right) D(x) x-\left(\begin{array}{l}
n-4 \\
n-5
\end{array}\right)\left(\begin{array}{l}
3 \\
3
\end{array}\right) x D(x) \\
& -\left(\begin{array}{l}
n-4 \\
n-4
\end{array}\right)\left(\begin{array}{l}
3 \\
2
\end{array}\right) D(x) x-\cdots-\left(\begin{array}{l}
2 \\
1
\end{array}\right)\left(\begin{array}{l}
n-3 \\
n-3
\end{array}\right) x D(x)-\left(\begin{array}{l}
2 \\
2
\end{array}\right)\left(\begin{array}{l}
n-3 \\
n-4
\end{array}\right) D(x) x \\
& -\left(\begin{array}{l}
n-2 \\
n-2
\end{array}\right) x D(x)-\left(\begin{array}{l}
n-2 \\
n-3
\end{array}\right) D(x) x-\left(\begin{array}{l}
n-1 \\
n-2
\end{array}\right) D(x) x=0, \quad x \in R .
\end{aligned}
$$

The above equation reduces to

$$
\begin{aligned}
\frac{(n-1) n}{2} D\left(x^{2}\right)= & (n-1) x D(x)+(n-2) x D(x)+D(x) x+(n-3) x D(x) \\
& +2 D(x) x+(n-4) x D(x)+3 D(x) x+\cdots+2 x D(x) \\
& +(n-3) D(x) x+x D(x)+(n-2) D(x) x+(n-1) D(x) x, \quad x \in R .
\end{aligned}
$$

Thus, we have

$$
\frac{(n-1) n}{2} D\left(x^{2}\right)=\left(\sum_{i=1}^{n-1} i\right)(D(x) x+x D(x)), \quad x \in R .
$$

Since $R$ is $n$ !-torsion-free, it follows from the relation (9) that

$$
D\left(x^{2}\right)=D(x) x+x D(x), \quad x \in R
$$

In other words, $D$ is a Jordan derivation. As we have already mentioned, any Jordan derivation on a 2-torsion-free semiprime ring is a derivation. The proof of the theorem is complete.

It would be interesting to know whether the theorem can be proved without assuming that $R$ has identity. We conclude with the following conjecture.

Conjecture 2. Let $R$ be a semiprime ring with suitable torsion restrictions. Suppose that there exists an additive mapping $D: R \rightarrow R$ such that

$$
D\left(x^{n}\right)=\sum_{j=1}^{n} x^{n-j} D(x) x^{j-1}
$$

is fulfilled for all $x \in R$ and some integer $n>1$. In this case, $D$ is a derivation. 
3350 A note on derivations in semiprime rings

\section{Acknowledgment}

This research has been supported by the Research Council of Slovenia.

\section{References}

[1] M. Brešar, Jordan derivations on semiprime rings, Proc. Amer. Math. Soc. 104 (1988), no. 4, 1003-1006.

[2] J Jordan mappings of semiprime rings, J. Algebra 127 (1989), no. 1, 218-228.

[3] M. Brešar and J. Vukman, Jordan derivations on prime rings, Bull. Austral. Math. Soc. 37 (1988), no. 3, 321-322.

[4] D. S. Bridges and J. Bergen, On the derivation of $x^{n}$ in a ring, Proc. Amer. Math. Soc. 90 (1984), no. $1,25-29$.

[5] J. M. Cusack, Jordan derivations on rings, Proc. Amer. Math. Soc. 53 (1975), no. 2, 321-324.

[6] I. N. Herstein, Jordan derivations of prime rings, Proc. Amer. Math. Soc. 8 (1957), no. 6, 11041110.

[7] J. Vukman, On derivations of standard operator algebras and semisimple $H^{*}$-algebras, preprint, 2005.

[8] J. Vukman and I. Kosi-Ulbl, On derivations in rings with involution, Int. Math. J. 6 (2005), no. 2, $81-91$.

[9] - On some equations related to derivations in rings, Int.J. Math. Math. Sci. 17 (2005), 2703-2710.

[10] Equations related to derivations on standard operator algebras and semisimple $H^{*}$ algebras, preprint, 2005.

Joso Vukman: Department of Mathematics, Faculty of Education, University of Maribor, Koroška Cesta 160, 2000 Maribor, Slovenia

E-mail address: joso.vukman@uni-mb.si

Irena Kosi-Ulbl: Department of Mathematics, Faculty of Education, University of Maribor, Koroška Cesta 160, 2000 Maribor, Slovenia

E-mail address: irena.kosi@uni-mb.si 


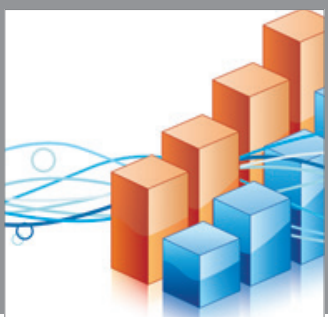

Advances in

Operations Research

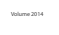

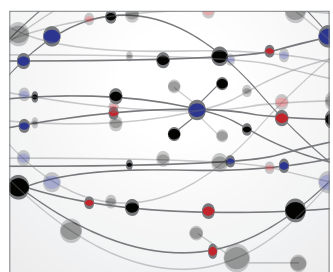

\section{The Scientific} World Journal
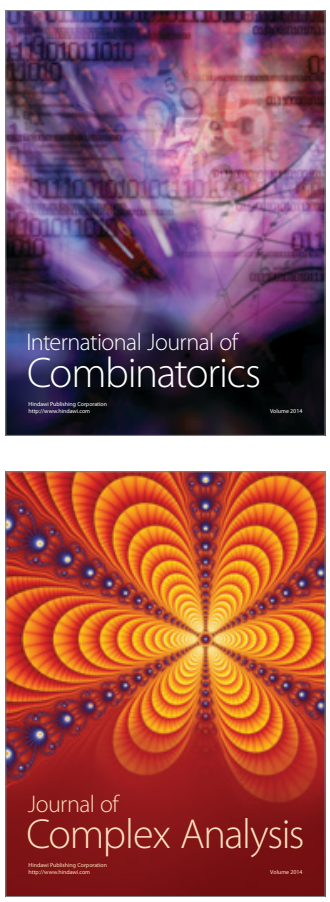

International Journal of

Mathematics and

Mathematical

Sciences
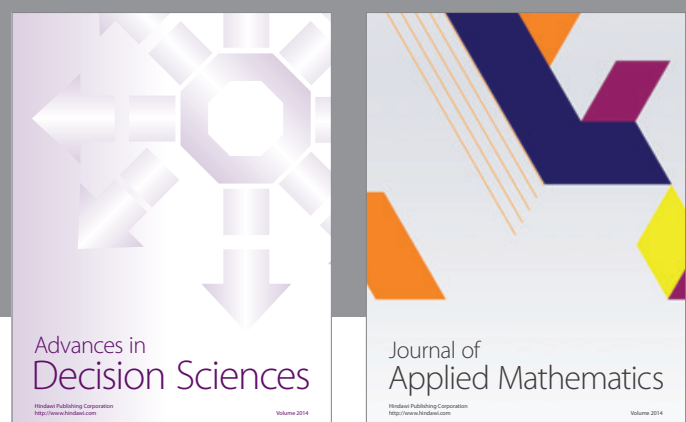

Journal of

Applied Mathematics
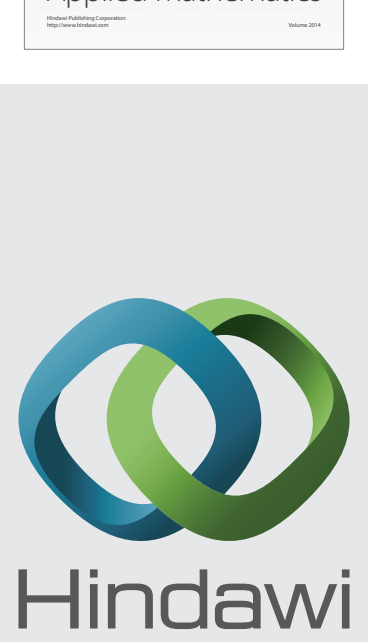

Submit your manuscripts at http://www.hindawi.com
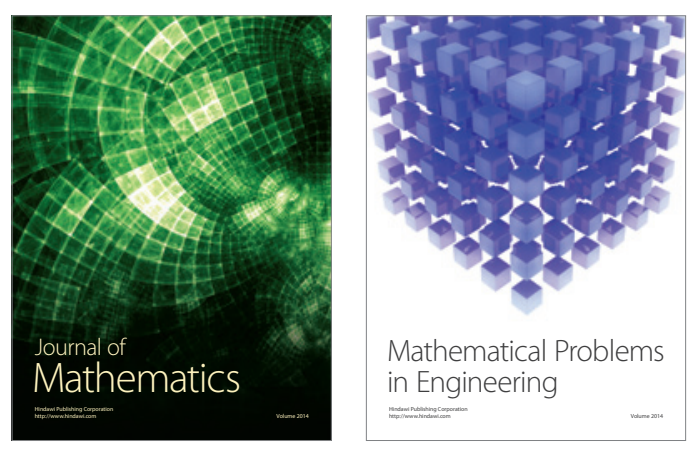

Mathematical Problems in Engineering
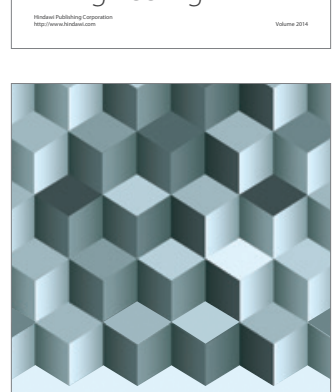

Journal of

Function Spaces
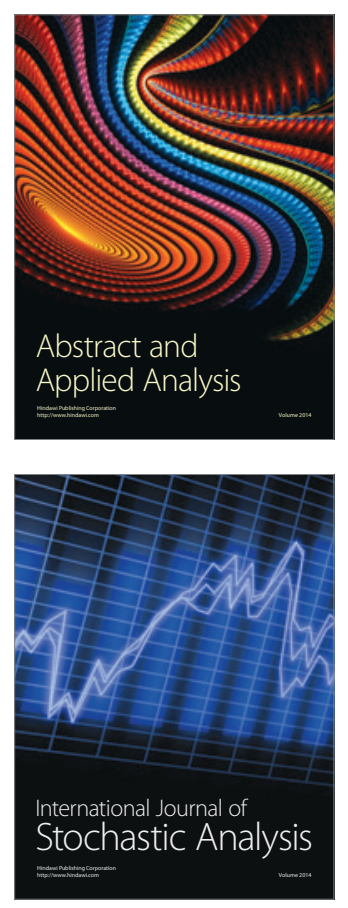

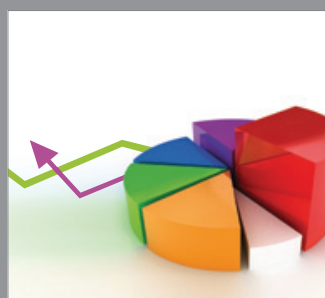

ournal of

Probability and Statistics

Promensencen
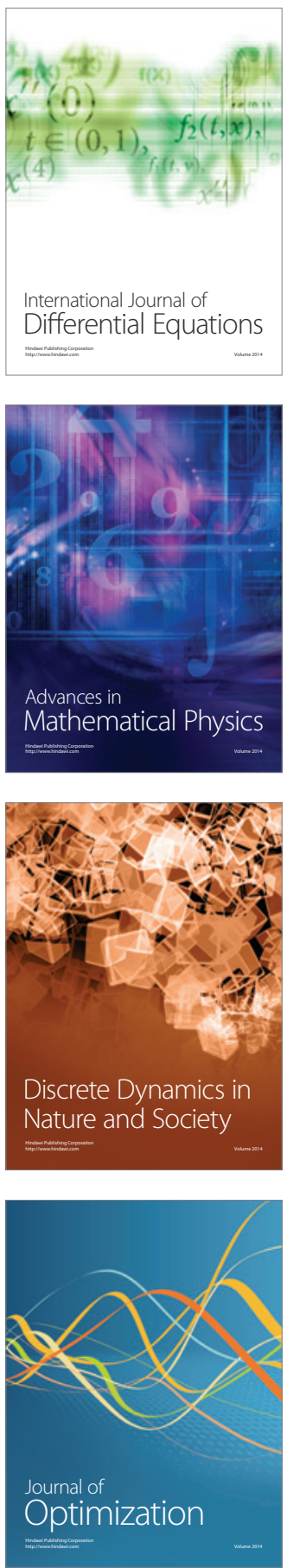